\title{
Syndrome coronarien aigu consécutif à un hématome spontané coronaire compressif
}

\author{
Acute Coronary Syndrome Related to a Spontaneous Coronary Intramural Compressive \\ Hematoma
}

\author{
M. Chabannon · G. Cayla $\cdot$ J.-E. de La Coussaye \\ Reçu le 8 septembre 2018; accepté le 11 février 2019 \\ (C) SFMU et Lavoisier SAS 2019
}

\section{Introduction}

Les hématomes intramuraux des artères coronaires sont une entité méconnue et sous-diagnostiquée de syndrome coronarien aigu (SCA). C'est un sous-type particulier de dissection des artères coronaires pouvant être iatrogène, traumatique ou spontanée. Nous présentons le cas d'une femme de 65 ans admise pour une douleur thoracique révélant un hématome pariétal compressif coronaire, compliqué au $12^{\mathrm{e}}$ jour d'un arrêt cardiorespiratoire (ACR).

\section{Observation}

Une patiente de 65 ans, sans facteur de risque cardiovasculaire (FDRCV), se présente aux urgences pour un premier épisode de douleur thoracique rétrosternale isolée, survenue brutalement au repos, oppressante, irradiant dans les deux bras et persistante depuis deux heures, sans contexte traumatique ou infectieux. L'examen clinique est normal avec $120 / 80 \mathrm{mmHg}, 55$ battements/minute et une saturation à $99 \%$ en air ambiant. L'électrocardiogramme (ECG) inscrit un rythme sinusal, avec des complexes QRS polymorphes (Fig. 1A) : alternance de QRS fins avec une sus-élévation du segment ST en inférolatéral ; extrasystoles ventriculaires ; QRS présentant un aspect de bloc de branche gauche. L'échographie transthoracique (ETT) retrouve une fraction

M. Chabannon $(\varangle) \cdot$ J.-E. de La Coussaye

Pôle anesthésie-réanimation douleur urgences,

CHU de Nîmes, F-30000 Nîmes, France

e-mail : margauxchabannon@hotmail.fr

G. Cayla

Cardiologie, CHU de Nîmes, F-30000 Nîmes, France

G. Cayla $\cdot$ J.-E. de La Coussaye

Université Montpellier, 2, rue École-de-Médecine,

F-34060 Montpellier, France d'éjection du ventricule gauche (FeVG) altérée à $40 \%$ avec une hypokinésie inféroseptoapicale, antéroseptale et antérolatéroapicale. Le diagnostic évoqué étant celui d'un SCA par rupture de plaque, la patiente reçoit aspirine et énoxaparine intraveineux. La coronarographie (Fig. 1B), réalisée en urgence, montre un aspect d'hématome intrapariétal de l'artère interventriculaire antérieure (IVA) moyenne avec occlusion de l'IVA distale. Un traitement conservateur est décidé avec aspirine $75 \mathrm{mg} / \mathrm{j}$ et bêtabloquants en soins intensifs cardiologiques. Le pic de troponine est atteint au cinquième jour à $1965 \mathrm{pg} / \mathrm{ml}$, permettant son retour à domicile le lendemain. Au dixième jour, elle présente un malaise avec sensation de mort imminente motivant sa réhospitalisation. Au $12^{\mathrm{e}}$ jour, elle fait une tachycardie ventriculaire à $260 / \mathrm{min}$ avec arrêt cardiocirculatoire, réduite par choc électrique externe. L'ECG post-ACR inscrit un bloc de branche gauche complet avec des ondes $\mathrm{T}$ négatives en antérieur. L'ETT après ACR retrouve une $\mathrm{FeVG}$ à $30 \%$ avec un anévrisme apical, une akinésie antéroapicale. Le contrôle coronarographique réalisé au décours immédiat objective une reperméabilisation complète de l'IVA distale avec un aspect persistant d'hématome intrapariétal et une longue sténose serrée de l'IVA moyenne et distale avec flux de compétition en distalité probablement responsable de l'ACR. Un défibrillateur automatisé interne est implanté, les bêtabloquants sont majorés et l'amiodarone introduite. L'échographie à un mois montre toujours une akinésie apicale avec un anévrisme apical et une FeVG altérée à $44 \%$. Il s'agit donc d'une patiente de 65 ans sans FDRCV ayant présenté un SCA de type I sur dissection spontanée de l'IVA compliquée d'une altération de la fonction ventriculaire gauche avec troubles du rythme.

\section{Discussion}

Les dissections spontanées des artères coronaires (SCAD) sont des pathologies rares, sous-diagnostiquées, notamment 


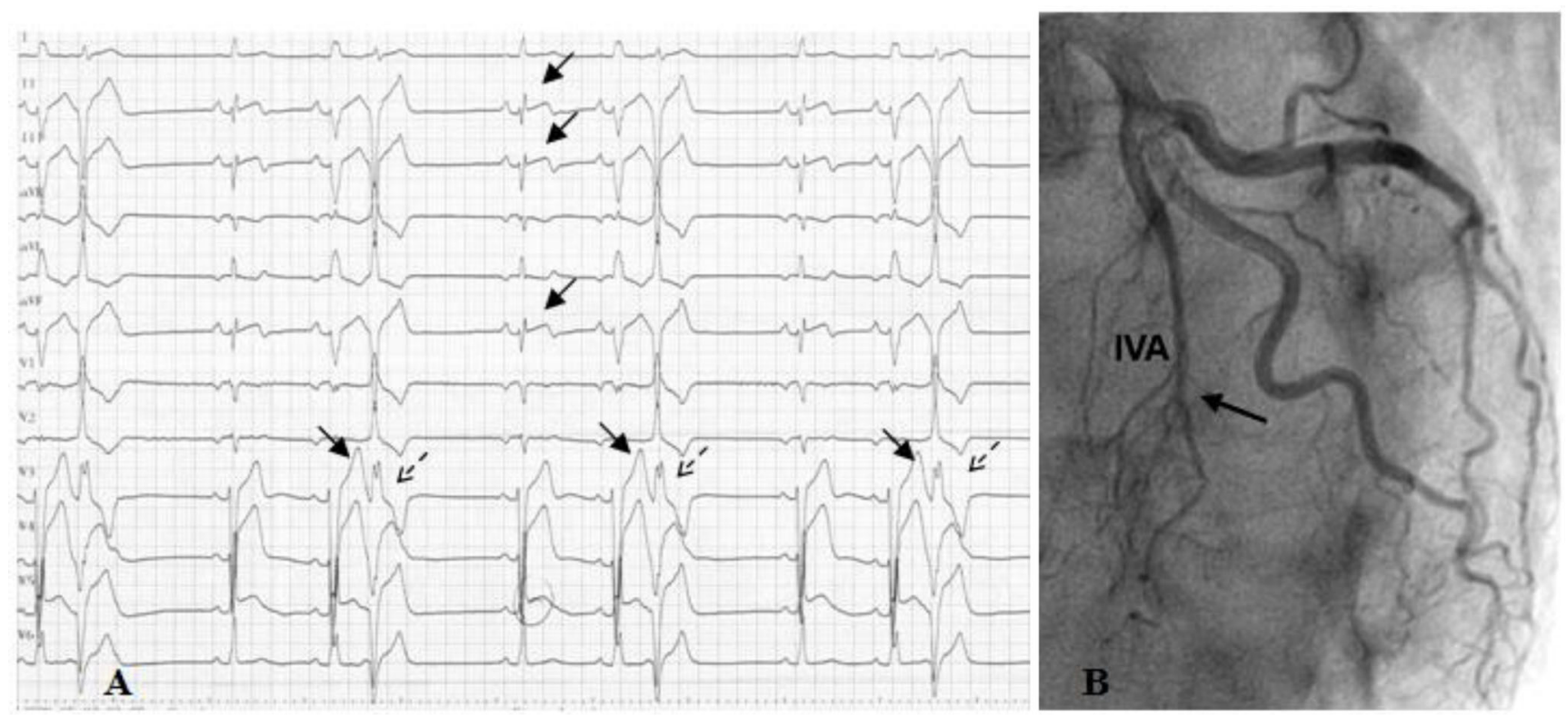

Fig. 1 A. Électrocardiogramme 12 dérivations montrant des QRS polymorphes : une sus-élévation du segment ST en inférolatéral ( $\boldsymbol{K}$ ), des extrasystoles ventriculaires $(\boldsymbol{K})$, un bloc de branche gauche $(\boldsymbol{y})$. B. Coronarographie montrant une longue sténose serrée de l'IVA moyenne avec occlusion de l'IVA distale

chez les femmes jeunes sans FDRCV [1-3]. Elles seraient responsables de plus de $10 \%$ des SCA avec sus-élévation du segment ST des femmes et jusqu'à $25 \%$ de tous les SCA avec élévation de troponine chez les femmes de moins de 50 ans [4]. L'âge moyen varie de 44 à 53 ans dans les séries contemporaines, avec un sex-ratio d'un homme pour neuf femmes. Les hématomes intramuraux coronaires sont des dissections de la média avec formation d'une fausse lumière, sans déchirure intimale $[1-3,5,6]$, classé dans les infarctus de type II. La compression de la vraie lumière vasculaire par l'hématome pariétal entraîne une ischémie myocardique. La physiopathologie du SCAD reste inconnue [7]. Les maladies du tissu conjonctif, les dysplasies fibromusculaires, les maladies inflammatoires systémiques, la grossesse et le postpartum sont des facteurs prédisposant par faiblesse de la paroi artérielle $[1,4,5,7]$. Le stress émotionnel, la prise de toxiques (cocaïne), l'exercice physique intense, le tabagisme actif seraient des facteurs favorisants par augmentation des contraintes pariétales $[1,2,7]$. Cliniquement, un hématome pariétal coronaire se manifeste principalement par une douleur thoracique [2,5-8]. Cependant, la symptomatologie peut varier d'une absence de symptôme à un choc cardiogénique ou décès $[3,4,8,9]$. L'ECG révèle un SCA avec ou sans élévation du segment ST $[2,3,8]$ associé à des troubles du rythme ventriculaire dans 3 à $10 \%$ [7]. Le diagnostic est donc souvent différé et les patients traités comme des SCA par rupture de plaque [7] avec potentiellement des effets secondaires néfastes induits par le traitement médical initial. L'angiographie coronaire permet le diagnostic [9]. L'imagerie endocoro- naire (IVUS ou OCT) permet de fournir des informations concernant la taille du vaisseau et la longueur de l'hématome $[2,5,7,10]$. La diffusion de l'imagerie a permis un meilleur diagnostic de cette pathologie, car elle permet, lorsqu'elle est faisable, une très bonne visualisation de l'hématome intrapariétal $[1,8,10]$. Il n'existe pas de recommandations thérapeutiques formelles [1-9]. La pratique actuelle préconise une mono- ou biantiagrégation plaquettaire du fait d'une fréquente association d'une dissection avec un thrombus intracoronaire $[4,7]$. En cas de dysfonction du ventricule gauche, les recommandations actuelles suivent celles des SCA avec des bêtabloquants et des inhibiteurs de l'enzyme de conversion de l'angiotensine à doses maximales [7]. Les anticoagulants ne sont recommandés qu'en cas d'angioplastie, et la thrombolyse contre-indiquée par leur risque hémorragique [7]. La cicatrisation vasculaire est obtenue dans la plupart des cas spontanément en quelques mois [2,6], et la revascularisation ne réduit pas le risque de récidive ou de complication à distance [3]. Le traitement par revascularisation des SCAD est difficile du fait d'une paroi vasculaire coronaire perturbée et fragile. Ce traitement devrait donc être restreint aux patients présentant une instabilité hémodynamique [1,2,7]. L'évolution initiale est en général bonne avec une mortalité hospitalière inférieure à $5 \%[1,2,4,9]$.

En conclusion, l'hématome pariétal coronaire spontané compressif est une pathologie méconnue qui doit être suspectée devant une douleur thoracique chez une femme jeune sans FDRCV ou d'un patient présentant une dysplasie fibromusculaire. 
Liens d'intérêts : les auteurs déclarent ne pas avoir de liens d'intérêts.

\section{Références}

1. Malcles G, Combaret N, Motreff P (2015) Spontaneous coronary artery dissection and ST-segment elevation myocardial infarction (STEMI). Ann Cardiol Angeiol 64:467-71

2. Antonsen L, Thayssen P, Jensen L (2015) Large coronary intramural hematomas: a case series and focused literature review. Cardiovasc Revasc Med 16:116-23

3. Tweet MS, Eleid MF, Best PJM, et al (2014) Spontaneous coronary artery dissection: revascularization versus conservative therapy. Circ Cardiovase Interv 7:777-86

4. Vanzetto G, Berger-Coz E, Barone-Rochette G, et al (2009) Prevalence, therapeutic management and medium-term prognosis of spontaneous coronary artery dissection: results from a database of 11,605 patients. Eur J Cardiothorac Surg 35:250-4
5. Raja Y, Trevelyan J, Doshi SN (2012) Intramural hemotoma presenting as acute coronary syndrome: the importance of intravascular ultrasound. Cardiol J 19:323-5

6. Ikegami R, Tsuchida K, Oda, H (2012) Acute myocardial infarction caused by spontaneous coronary intramural hematoma. J Invasive Cardiol 24:692-3

7. Adlam D, Alfonso F, Maas A, et al (2018) European society of cardiology, acute cardiovascular care association, SCAD study group: a position paper on spontaneous coronary artery dissection. Eur Heart J 39:3353-68

8. Hsieh YC, Tsai IC, Tsao CR, et al (2010) Spontaneous remission of ruptured intramyocardial hematoma detected upon serial multidetector computed tomography. Tex Heart Inst J 37:350-3

9. Alfonso F, Paulo M, Lennie V, et al (2012) Spontaneous coronary artery dissection: long-term follow-up of a large series of patients prospectively managed with a "conservative" therapeutic strategy. JACC Cardiovasc Interv 5:1062-70

10. Akodad M, Lattuca B, Ledermann B, et al (2015) Optical coherence tomography evidence of spontaneous healing of an intramural coronary hematoma. Can J Cardiol 31:820 